\title{
The assessment of motor control in sighting dominance using an illusion decrement procedure
}

\author{
CLARE PORAC \\ University of Victoria, Victoria, British Columbia, Canada V8W 2 Y2 \\ and \\ STANLEY COREN \\ University of British Columbia, Vancouver, British Columbia, Canada V6T IW5
}

\begin{abstract}
Walls (1951) proposed that perceptual asymmetries between the sighting dominant and the nonsighting dominant eyes were based upon differences in the monitoring of eye movements. The present research explored this hypothesis in the context of an illusion decrement paradigm. Since illusion decrement seems to occur only under conditions of free eye-movement inspection, it was reasoned that any motoric asymmetries would manifest themselves through differences in the rate and extent of decrement. These predictions were partially confirmed. The sighting eye manifested greater illusion decrement, but the effect remained specific to conditions where both eyes were stimulated. In addition, asymmetries in the interocular transfer of illusion decrement were found to favor the sighting dominant eye.
\end{abstract}

Eye dominance is a term which has been used to describe the preferential use of information from one eye in a variety of visual coordinations (Porac \& Coren, 1976). The form of eye dominance most frequently mentioned is sighting dominance. ${ }^{1}$ Sighting dominance can be observed in tasks where an individual is required to align a near target with a more distant target when both eyes are opened. It is difficult to accomplish such an alignment since only one of the two targets can be fixated at any one time. The fixated target (whether near or far) will be fused binocularly, but the nonfixated target will stimulate noncorresponding retinal points, giving rise to diplopia. A conscious strategy to deal with these double images would be to close one eye. The unconscious analog to this strategy involves the temporary suppression of the stimulation to one eye until the alignment has been completed. The nondominant or nonsighting eye is suppressed while the sighting or dominant eye takes over to perform the visual task.

A number of reports indicate that approximately $65 \%$ of all visually normal individuals consistently use their right eyes and suppress their left eyes in performing alignment tasks (Coren \& Kaplan, 1973; Duke-Elder, 1949; Porac \& Coren, 1975, 1976). Thus, approximately two-thirds of any population will be

We would like to acknowledge the assistance of Patricia and Fred $W$. Whitford in the collection of the data and the employees of the Royal Trust of Canada (Victoria, B.C., branch office), who contributed their time to serve as subjects. We also acknowledge research support from the National Research Council of Canada, Grants A0293 and A9783, and from the Medical Research Council of Canada, Grant MA-5382.

right-eyed dominant while the remaining one-third will be left-eyed. These percentages are consistent and appear to be developmentally stable from infancy through to adulthood (Coren, 1974). They also appear to be independent of cultural influence (Porac \& Coren, 1976).

Although investigators have been aware of the existence of eye dominance for centuries (Porta, 1593), little is known about the mechanisms which underlie it. The most recent attempt to formulate a theoretical account of eye dominance was reported 25 years ago by Walls (1951). He suggested that information about the relative egocentric direction of visual targets was determined by the line of sight of the sighting dominant eye, rather than in reference to the medial sagittal plane of the head or a cyclopean eye. Furthermore, he maintained that the movements of the dominant eye are monitored by the visual system in order to ascertain target locus while the movements of the nondominant eye are ignored. Empirical results have provided some support for Walls' hypothesis. Several investigators have confirmed the prediction that the egocentric "straight ahead" is shifted towards the side of the dominant eye (Foley \& Held, 1972; Howard \& Templeton, 1966; Ogle, 1962). Others have indicated some degree of motor dominance of the sighting eye as required by the theory. For instance, the sighting eye appears to possess more movement accuracy (Clark, 1935) and fixation stability (Schoen \& Schofield, 1935) than the nonsighting eye. Money (1972) reported performance superiority for the sighting eye in tasks involving eye movements, and no superiority when the eyes were stationary. 
After a quarter of a century, it is time to reconsider the essence of Walls' theory which maintains that the two eyes receive symmetrical motor commands which are then asymmetrically monitored and employed in the construction of the final percept. Since the primary information about eye movements comes from the sighting dominant eye, one should find that the motoric information from this eye is "better" or more accurate than that from the nondominant eye. However, there are difficulties in testing this notion directly. One must engage the observer in a task which requires eye movements and also the opportunity to ascertain how movement information from each eye is utilized in the construction of the percept. A procedure which fulfills this requirement is suggested in the visual illusion literature.

In 1896, Heymans reported that the magnitude of the Mueller-Lyer illusion decreased with prolonged exposure to the figure. Since that time, this finding has been replicated many times with the Mueller-Lyer illusion (Coren \& Girgus, 1972a, b, 1974; Dewar, 1968; Festinger, White, \& Allyn, 1968; Girgus, Coren, Durant, \& Porac, 1975; Girgus, Coren, \& Horowitz, 1973; Judd, 1902; Mountjoy, 1958), and with other illusion figures as well (Coren \& Girgus, 1972b). A prerequisite for the diminution of illusion magnitude seems to be the opportunity to freely inspect the illusory configuration with saccadic eye movements. Little illusion decrement occurs in the absence of free eye-movement exploration (Burnham, 1968; Coren \& Hoenig, 1972; Festinger et al., 1968; Hoenig, 1972). Attempts have been made to investigate the relationship between saccadic eye movements and illusion decrement by recording fixation patterns. It has been noted that when the observer tries to fixate a vertex on the apparently longer portion of the Mueller-Lyer, he emits eye movements which tend to be too long. This "overshoot" by the eye necessitates a corrective saccadic flick back toward the vertex. The converse occurs when an observer inspects the apparently shorter segment, where an initial undershoot is followed by a corrective movement outward (Festinger et al., 1968). It has been suggested that the extent and the direction of such corrective movements provide information about the nature, magnitude, and direction of the illusory distortion. These data are then used to correct the erroneous percept (Burnham, 1968; Coren \& Hoenig, 1972). In line with this explanation, Festinger et al. (1968) reported that continued inspection caused decreases in illusion magnitude and concommitantly increases in the accuracy of saccadic movements over the figure.

The utilization of feedback information from both eye movements and eye-movement corrections would seem to depend upon some means of monitoring the extent and direction of the movements which are emitted. If Walls (1951) is correct, one would expect that such movement feedback is most efficient for the sighting eye. Not only should there be greater monitoring efficiency, but the theory implies that the information itself should be better and more readily available. As a method to test this theory, it seems reasonable to assume that one could use the rate and the extent of illusion decrement, under conditions of free inspection, to investigate differences between the dominant and the nondominant eye in the degree of motoric efficiency and control. These considerations form the basis of the present investigation.

\section{METHOD}

\section{Stimulus and Apparatus}

The Brentano version of the Mueller-Lyer figure was employed. The apparently longer portion of the illusion was made adjustable by means of a tongue and groove arrangement. The length of the fixed shaft was $8 \mathrm{~cm}$; the length of the wings was $2 \mathrm{~cm}$ and formed an angle of $45^{\circ}$ with the horizontal. Illusion magnitude was measured in millimeters from a scale attached to the adjustable portion of the stimulus.

In addition to a monocular viewing situation where the contralateral eye is occluded while the action of one eye is observed, an attempt was made to study the action of each eye singly under conditions which allowed for almost complete binocularity. The present study employed two types of viewing conditions, both of which permitted the study of each eye separately. The first was similar to a procedure which has been called equivalence occlusion (Lehmkuhle \& Fox, 1976). Both eyes are open and receiving equivalent illumination and pattern information; however, the critical stimulus (in this case, the illusion configuration) is presented to only one eye. A technique analogous to that used in anaglypic viewing was employed to accomplish this end. The subjects wore a pair of goggles which held a red filter in front of one eye and a green filter in front of the other. The filters were matched for transmittance with approximately $42 \%$ of the incident light transmitted through each filter. The illusion configuration was drawn in a red ink which matched the spectral transmittance of the red filter. With the goggles in front of the eyes, the figure was invisible through the red channel but appeared to be drawn in black lines to the eye which wore the green filter. The lateral field of view was limited to the central $29^{\circ}$ so that portions of the visual field usually seen monocularly were eliminated and the available field of view for the two eyes completely overlapped. Since all of the contours in the environment, except for the illusion figure, were available to both eyes, this condition is referred to as the binocular viewing situation. The goggles were constructed so that filters could be switched from one eye to the other. In addition, it was possible to replace the red filter with an opaque occluder. As mentioned, monocular occlusion is the more common technique for studying the action of one eye, since it eliminates all pattern and brightness information from the eye not under investigation. In this experiment, opaque occlusion of one eye was also utilized, and this condition has been called monocular viewing.

At the viewing distance used in the experiment, the fixed shaft of the illusion figure subtended a visual angle of $11^{\circ}$. Illumination, provided by a tungsten source, was approximately $800 \mathrm{~lx}$.

\section{Subjects}

Eighty adult volunteers participated as observers. All subjects underwent a visual screening using portions of the Keystone Visual Skills Battery. Only subjects with uncorrected monocular acuity of $20 / 30$ or better (tested at both near and far convergence distances) participated in the experiment. 
The sighting dominance of all observers was tested using two different indices. The first, called the Point Test, requires the observer to point to the experimenter's nose while keeping both eyes opened. The experimenter, who stands approximately $2 \mathrm{~m}$ in front of the subject, notes which eye is aligned with the outstretched finger. The observer points alternately with the right and left hands to control for manual dominance bias. The second procedure for assessing eye dominance was the ABC Test. The observer holds the wide end of a truncated cone close to the face and views a target through the smaller of the two apertures while keeping both eyes open. The cone must be squeezed between the hands in order to be held open. The eye performing the alignment can be seen through the aperture and scored as the dominant eye. These procedures have been shown to be reliable indicators of the sighting dominant eye (Coren \& Kaplan, 1973; Crider, 1944; Gronwall \& Sampson, 1971; Miles, 1929; Porac \& Coren, 1975, 1976). Each test was administered four times and, on the basis of the composite score from these two tests, the subjects were classified as either right- or left-eyed sighters. The final sample consisted of $\mathbf{4 0}$ right-sighters and $\mathbf{4 0}$ left-sighters. All observers were naive as to the purpose of the experiment.

\section{Procedure}

Decrement training. Twenty right-eyed and 20 left-eyed observers were assigned to the binocular viewing condition. Ten right-sighters and 10 left-sighters viewed the illusion with their dominant eyes, while the remainder inspected the illusion with their nondominant eyes. A similar assignment was used for the monocular viewing group.

The decrement procedure was similar to one which has been used previously (Coren \& Girgus, 1972a, b, 1974; Girgus et al., 1973; Girgus et al., 1975). This method leads to sizable reductions in illusion magnitude. At the outset, each observer set the two portions of the illusion figure to apparent equality. This was followed by 5 min of inspection with free eye movements in which the subject was instructed to move the eyes across the figure, fixating each of the three vertices in turn (the configuration was set to physical equality during this inspection). Settings to apparent equality were taken at 1 -min intervals. The eye-movement instructions were repeated after each of the measurements of illusion magnitude, and subjects appeared to have no difficulty in following these directions.

Measurement of interocular transfer. The rate and extent of illusion decrement can provide information about the efficiency of the motoric feedback system and any asymmetries related to eye dominance. However, it was felt that differences in monitoring or the salience of movement information from the sighting and the nonsighting eyes could be explored more fully using an interocular transfer procedure. Previous research has demonstrated greater interocular transfer of tilt and motion aftereffects when the sighting eye is exposed to the inducing stimuli (Mitchell, Reardon, \& Muir, 1975; Mitchell \& Ware, 1974; Movshon, Chambers, \& Blakemore, 1972; Wade, 1976). Given such evidence for asymmetries between the eyes, it seemed useful to extend such testing to the decrement paradigm.

Interocular transfer measures were obtained by taking settings of illusion magnitude on the unexposed eye, that is, the eye which would not undergo decrement. This was done immediately prior to and immediately following the decrement training. Any reduction in illusion magnitude from the pretest to the posttest measurement on the unexposed eye was taken as evidence of interocular transfer of illusion decrement.

\section{RESULTS}

Separate analyses of right- and left-eyed sighters revealed no systematic differences between these subjects; therefore, all subsequent analyses were collapsed across this variable. The following data are presented in terms of the sighting vs. the nonsighting eye.

\section{Illusion Decrement}

Figure 1 contains a plot of illusion magnitude as a function of inspection time. As expected, both the binocular and the monocular exposure groups showed significant reduction of illusion strength during the course of $5 \mathrm{~min}$ of free viewing, $F(5,190)$ $=19.14, \mathrm{p}<.001$, for binocular viewing, and $F(5,190)=25.75, p<.001$, for monocular viewing. This simply verifies that illusion decrement had taken place. There were, however, differences as a function of the viewing condition and the eye used during the inspection.

In the monocular viewing group, there was significant illusion decrement when either the dominant or nondominant eye was used. Illusion magnitude was reduced in both eyes to about the same degree, and there were no significant differences in the amounts of illusion decrease, $F(1,38)=1.13$. The pattern was different for the binocular viewing condition. As Figure 1 shows, there was greater reduction in illusion magnitude when the configuration was inspected by the sighting eye, $F(1,38)=7.84, p<.01$.

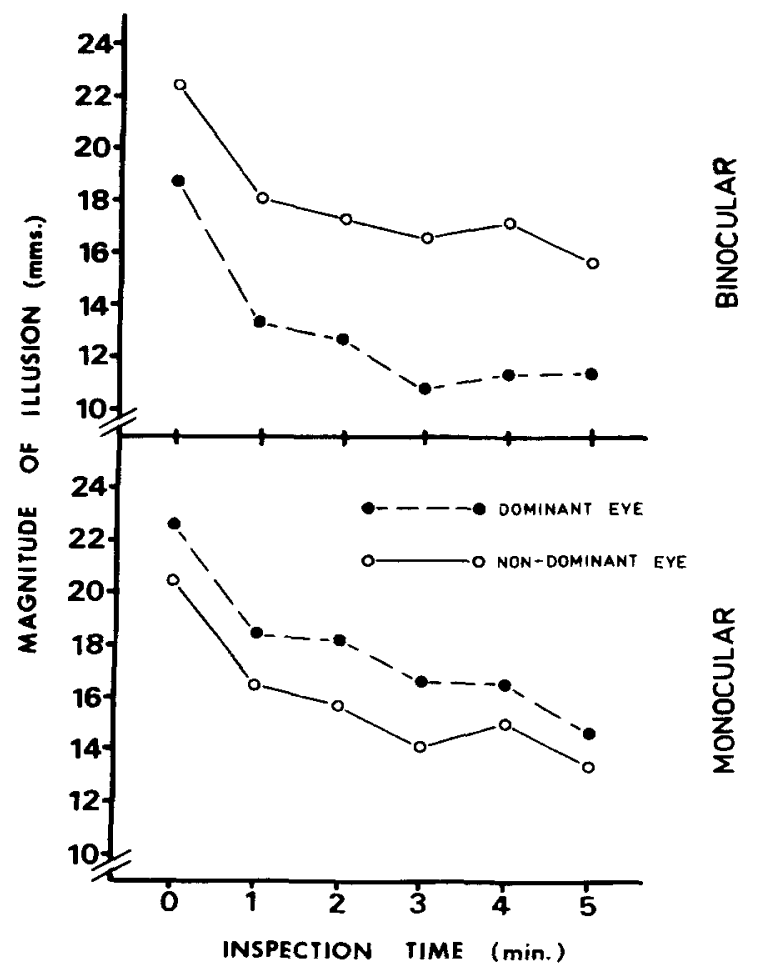

Figure 1. Reduction in illusion magnitude over $5 \mathrm{~min}$ of free viewing as a function of whether the dominant or the nondominant eye was used. Top: Illusion decrement under binocular viewing (equivalence occlusion). Bottom: Illusion decrement under monocular viewing (opaque occlusion). 
Thus, although there was no significant difference between the sighting and the nonsighting eyes prior to inspection, $F(1,38)=2.90$, after $5 \mathrm{~min}$ of free viewing the sighting eye showed a significantly smaller illusion, $F(1,38)=8.69, p<.01$. The reduction amounted to $42 \%$ after $5 \mathrm{~min}$ of inspection by the sighting eye, while a $22 \%$ decrease was found for the nonsighting eye. Although both eyes functioned with approximate equality under monocular exposure (both accomplishing about $35 \%$ reduction in illusion magnitude), the ability of the nonsighting eye to overcome the illusory distortion by scanning the Mueller-Lyer figure appeared to be reduced by $13 \%$ when visual information was processed by both eyes.

\section{Interocular Transfer}

As a measure of the interocular transfer of illusion decrement, the final postinspection illusion magnitude of the unexposed eye was subtracted from the preinspection response on the same eye. This pretest/posttest difference score is a measure of the change in illusion magnitude which results from the exposure of the contralateral eye to the figure. The results are shown in Figure 2.

Interocular transfer was relatively symmetrical in the monocular viewing condition. There was no difference in the amount of transfer as a function of the eye that was used to inspect the illusion $F(1,38)=$ .04. The pattern was quite different in the binocular viewing situation. Here, there was significantly more interocular transfer when the dominant eye inspected the illusion configuration, $F(1,38)=7.85, p<.01$. These results appear to mimic the illusion decrement data. Again, the dominant and the nondominant eyes performed equally well under monocular exposure conditions, producing approximately equal amounts of interocular transfer. However, when both eyes were functioning, with the critical stimulus presented to only the eye under study, there was a performance difference which favored the sighting eye.

\section{DISCUSSION}

Burnham (1968), Coren and Hoenig (1972), and Festinger et al. (1968) have suggested that decrement of the Mueller-Lyer illusion depends upon a recalibration of eye movements occurring as a result of error information from saccades which are originally too long or too short as the eye moves across the figure. If this is the case, then the present results provide only partial support for Walls' theory of eye dominance. In its strongest form, the theory predicts that there should be no information about the extent and direction of ocular movement available to the nondominant eye. As Walls contends: "My thesis is that in any individual who has a motordominant eye an innervation record is kept only for

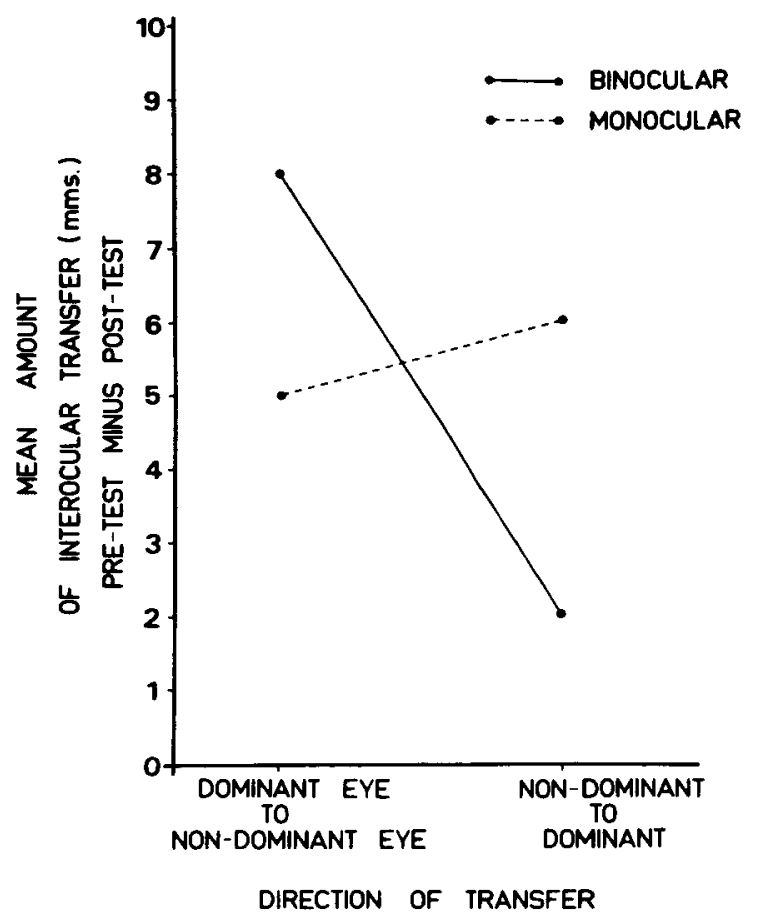

Figure 2. Interocular transfer of illusion decrement. The numbers represent the preinspection/postinspection difference scores as measured on the unexposed eye.

the muscles of that eye, that this is what motor dominance really means and that this explains all of the properties of the 'dominant' eye-both in binocular vision and so far as they differ from those of the other eye when each eye is used alone."

Based on this statement, one would expect that virtually no illusion decrement would occur in the nondominant eye, and one would also expect that the binocular and monocular viewing conditions would produce identical results. Clearly, this is not what occurred in the present experiment. The monocular situation produced identical results regardless of the dominance of the eye used. Under conditions of binocular viewing, however, the sighting eye accomplished significantly more decrement within the 5-min exposure period. Hence, the latter viewing condition is the only one which resulted in asymmetrical performance. In addition, the fact that illusion decrement occurred in the nonsighting eye under both viewing conditions is evidence that movement information from that eye is not completely ignored by the higher visual centers as Walls suggests. When both eyes are used simultaneously, there seems to be some attenuation in the utilization of movement information from the nonsighting eye. This attenuation does not occur unless the sighting eye is stimulated. Hence, there is some support for asymmetrical usefulness of eye-movement information from the sighting and the nonsighting eyes, but, since it only appears under conditions of binocular 
exposure, it is a relative, rather than an exclusive, asymmetry.

The interocular transfer data lead to a similar set of conclusions. When the sighting or the nonsighting dominant eye is tested monocularly, there is little difference in the amount of transfer of illusion decrement. When both eyes are functioning, but the illusion stimulus is only present in one, an asymmetry in transfer appears. When it is the sighting eye which has been exposed to the illusion, there is greater transfer of decrement than if the exposure has been to the nonsighting eye. Colthart (1973) has suggested that interocular transfer is mediated by binocularly driven cells in the visual cortex. The present results may indicate that binocular cortical cells are somewhat more responsive to input from the sighting eye. They certainly indicate that, under conditions of binocular exposure, the visual system weighs input from the sighting dominant eye more heavily in the construction of binocular percepts. These conclusions are consistent with those of the previously mentioned studies on interocular transfer of the tilt and motion aftereffects (Mitchell et al., 1975; Mitchell \& Ware, 1974; Movshon et al., 1972; Wade, 1976), and extend the superiority of transfer from the sighting to the nonsighting eye to illusion decrement as well.

Although these results do not fully support Walls' contentions, they provide additional information about the nature of ocular dominance. In a recent review, Porac and Coren (1976) suggested that eye dominance may be important for organisms that have large overlapping monocular fields of view. Such organisms must contend with the problem of diplopia in many common situations. For instance, in pointing a finger at a distant target, one cannot effectively use both eyes. Fixation on the fingertip will result in stimulation of noncorresponding retinal points by the distant target. Thus the target will appear diplopic. Fixation upon the target will give rise to double images of the fingertip. A behavioral strategy must be adopted to solve this problem, or else one is left with the problem of pointing to two phenomenal targets with one finger or to one target with two fingers. Porac and Coren (1976) suggest that the problem is solved by suppressing the input to the nonsighting eye. This clears the way for single vision by admitting only the input from the sighting eye. This habitual strategy gives rise to the consistency and reliability of sighting dominance behaviors.

There is some support for this type of reasoning in clinical data. Coren and Duckman (1975) looked at a large sample of individuals with strabismus. For these patients, one eye is deviated from the principal line of sight, and foveal stimulation is not congruent. Hence, there may be chronic complaints of diplopic vision. Often one observes a functional monocular suppression in such cases. This suppression is called amblyopia ex anopsia, which is a long-term adaptation that serves to eliminate the double vision by suppressing the input to one eye. Coren and Duckman (1975) found that amblyopic suppression is most apt to develop in the nonsighting eye. Within the context of such findings, the pattern of data in the present experiment indicates an attenuation or suppression of information from the nonsighting eye under conditions of binocular stimulation. To push the parallel between pathological suppression and the normal operation of eye dominance leads to the observation that amblyopic suppression often disappears when the visually normal eye is occluded and the amblyopic eye is tested monocularly (cf. Shapero, 1971). This is similar to the disappearance of performance asymmetries under conditions of monocular viewing which occurred in the present experiment. Such a similarity suggests that clinically observed cases of suppression and the normal suppressive coordination of the sighting and of the nonsighting eyes may be points on a continuum that is mediated by common mechanisms.

Although these results do not fully support Walls' hypothesis that motoric information is only monitored from the dominant eye, they do indicate that movement information from the nonsighting eye may be less effectively monitored than information from the sighting eye under conditions of binocular stimulation. Whether this difference is completely motoric in nature or involves higher level information processing asymmetries remains to be established. Regardless of the source, however, there are clear differences in the performance of the dominant and nondominant eyes.

\section{REFERENCES}

Burnham, C. A. Decrement in the Mueller-Lyer illusion with saccadic and tracking eye movements. Perception \& Psychophysics, $1968,3,424-426$.

CLARK, B. The effect of binocular imbalance on the behavior of the eyes during reading. Journal of Educational Psychology, 1935, 26, 530-538.

Colthart, $M$. Colour-specificity and monocularity in the visual cortex. Vision Research, 1973, 13, 2595-2598.

CoRen, S. The development of ocular dominance. Developmental Psychology, 1974, 10, 304.

CoRen, S., \& Duckman, R. Ocular dominance and amblyopia. American Journal of Optometry and Physiological Optics, 1975, 52, 47-50.

Coren, S., \& Girgus, J. S. Differentiation and decrement in the Mueller-Lyer illusion. Perception \& Psychophysics, 1972, 12, 466-470. (a)

Coren, S., \& Girgus, J. S. Illusion decrement in intersecting line figures. Psychonomic Science, 1972, 26, 108-110. (b)

Coren, S., \& GIRgus, J. S. Transfer of illusion decrement as a function of perceived similarity. Journal of Experimental Psychology, 1974, 10, 881-887. 
Coren, S., \& Hoenig, P. Eye movements and decrement in the Oppel-Kundt illusion. Perception \& Psychophysics, 1972, 12, 224-225.

Coren, S., \& Kaplan, C. P. Patterns of ocular dominance. American Journal of Optometry and Archives of the American Academy of Optometry, 1973, 50, 283-292.

CrIDER, B. A battery of tests for the dominant eye. Journal of General Psychology, 1944, 31, 179-190.

DEWAR, R. E. Distribution of practice and the Mueller-Lyer illusion. Perception \& Psychophysics, 1968, 3, 246-248.

DUKE-EDLER, W. S. Textbook of ophthalmology (Vol. 4). London: Henry Kimpton, 1949.

Festinger, L., White, C. W., \& Allyn, M. R. Eye movements and decrement in the Mueller-Lyer illusion. Perception \& Psychophysics, 1968, 3, 376-382.

FOLEY, J. E., \& HELD, R. Visually directed pointing as a function of target distance, direction and available cues. Perception \& Psychophysics, 1972, 12, 263-268.

Girgus, J. S., Coren, S., Durant, M., \& Porac, C. The assessment of components involved in illusion formation using a long term decrement procedure. Perception \& Psychophysics, 1975, 18, 144-148.

Girgus, J. S., Coren, S., \& Horowitz, L. Peripheral and central components in variants of the Mueller-Lyer illusion. Perception \& Psychophysics, 1973, 13, 157-160.

Gronwall, D. M., \& Sampson, H. Ocular dominance: A test of two hypotheses. British Journal of Psychology, 1971, 62, 175-185.

HeYmaNs, G. Quantitative Untersuchungen über das "optischen Paradoxen." Zeitschrift für Psychologie, 1896, 9, 221-255.

HoENIG, P. The effects of eye movements, fixation and figure size on decrement in the Mueller-Lyer illusion. Unpublished doctoral dissertation, Graduate Faculty of the New School for Social Research, 1972.

HowaRD, I. P., \& Templeton, W. B. Human spatial orientation. New York: Wiley, 1966.

JuDD, C. H. Practice and its effects on the perception of illusions. Psychological Review, 1902, 9, 27-39.

LEHMkUHIE, S. W., \& Fox, R. On measuring interocular transfer. Vision Research, 1976, 16, 428-430.

Miles, W. R. Ocular dominance demonstrated by unconscious sighting. Journal of Experimental Psychology, 1929, 12, 113-126.

Mitchell, D. E., Reardon, J., \& MuIR, D. W. Interocular transfer of the motion after-effect in normal and stereoblind observers. Experimental Brain Research, 1975, 22, 163-173.

MrtcheLl, D. E., \& WARE, C. Interocular transfer of a visual after-effect in normal and stereoblind humans. Journal of Physiology, 1974, 236, 207-221.

MoneY, J. Studies on the functioning of sighting dominance. Quarterly Journal of Experimental Psychology, 1972, 24, 454-464.

MounTJoy, P. T. Effects of exposure time and intertrial interval upon decrement to the Mueller-Lyer illusion. Journal of Experimental Psychology, 1958, 56, 97-102.

Movshon, J. A., Chambers, B. E. I., \& Blakemore, C. Interocular transfer in normal humans and those who lack stereopsis. Perception, 1972, 1, 483-490.

OGLE, K. N. Ocular dominance and binocular retinal rivalry. In H. Davson (Ed.), The eye (Vol. 4). New York: Academic Press, 1962.

Porac, C., \& Coren, S. Is sighting dominance a part of generalized laterality? Perceptual and Motor Skills, 1975, 40, 763-769.

Porac, C., \& Coren, S. The dominant eye. Psychological Bulletin, $1976,83,880-897$.

Porta, I. B. De refractione. Optics Parte: Libri Novem. Ex officina Horatij Salvania. Naples: Apud Io Iacobum Carlinum \& Anotinium Pacem 1593.

Schapero, M. Amblyopia. Philadelphia: Chilton, 1971.

Schoen, Z. J., \& Schofield, C. F. A study of the relative neuromuscular efficiency of the dominant and non-dominant eye in binocular vision. Joumal of General Psychology, 1935, 11, 156-181.

W ADE, N. On interocular transfer of the movement after-effects in individuals with and without normal binocular vision. Perception, 1976, 5, 113.118.

W Alls, G. L. A theory of ocular dominance. A.M.A. Archives of Ophthalmology, 1951, 45, 387-412.

\section{NOTE}

1. The terms eye dominance and sighting dominance are used interchangeably.

(Received for publication October 8, 1976; revision accepted February 4, 1977.) 